\title{
LA RESPONSABILIDAD SOCIAL
}

\author{
EMPRESARIAL: HERRAMIENTA ESTRATÉGICA \\ PARA LA COMPETITIVIDAD DE LAS PYMES \\ DE LA CADENA DE VALOR DE LA GRAN \\ EMPRESA DEL SECTOR TEXTIL
}

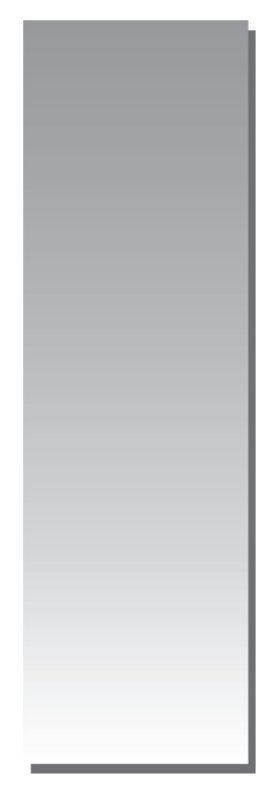

CORPORATE SOCIAL RESPONSIBILITY: STRATEGIC TOOL FOR COMPETITIVENESS OF SMES INCORPORATED INTO THE VALUE CHAIN OF LARGE TEXTILE COMPANY

\author{
Norka Patricia Stuart Alvarado* \\ pstuart@ulima.edu.pe \\ Andrea Robertha Aráuz Chávez ${ }^{* * *}$ \\ andrea.arauz@uleam.edu.pe
}

[RECEPCIÓN: ABRIL 2016 / CONFORMIDAD: MAYO 2016]

\section{RESUMEN}

El objetivo de la investigación es el de determinar en el marco de la responsabilidad social empresarial (RSE) las prácticas de negocio de la empresa basadas en valores éticos y principios de transparencia, las cuales buscan promover el mejoramiento permanente de la relación entre la empresa y sus partes interesadas (stakeholders).Estos segmentos incluyen a sus clientes, proveedores, socios, consumidores, medio ambiente, comunidades, gobierno y sociedad en general. La aplicación de la responsabilidad social empresarial (RSE) en la empresa tiene efectos en el clima organizacional, el desempeño operacional, capacidad de innovación y en la reputación de la empresa. La incorporación de las pymes que forman parte de la cadena de valor de la gran empresa, en la aplicación de la RSE se traducirá en una mejora de los procesos operativos, racionalización de estos procesos, reducción de costos y un mejor desempeño respecto al medio ambiente. Es la gran y mediana empresa la llamada incluir a las pymes en los proyectos y actividades mencionados, ya que dada la capacidad financiera de las pequeñas empresas frente a los costos en capacitación y certificación no sería viable el modelo de RSE. Esta aplicación de la RSE presenta una serie de desafíos estratégicos y operativos que pueden considerarse como oportunidades de desarrollo y mejora de los negocios, las cuales obligan a los gerentes a ser más creativos y responsables, a combinar una visión de la empresa no solo focalizada en el aumento de las utilidades y a incrementar las actividades en beneficio de sus proveedores, clientes y sociedad. La estrategia propuesta debe incentivar y facilitar que las empresas del sector textil y confecciones generen más empleo, que se relacionen mejor con las grandes empresas y entre sí, incorporen la mejor y más reciente tecnología, se adapten al nuevo modelo de especialización flexible, incrementen su creatividad y generen innovaciones tecnológicas y mejoren su competitividad.

Palabras clave: Competitividad; responsabilidad social empresarial; cadena de valor.

\footnotetext{
Doctora en Ciencias Administrativas por la Universidad Nacional Mayor de San Marcos. Master of Business Administration, California State University, Fullerton, EE.UU. Magíster en Administración de Negocios, Universidad San Ignacio de Loyola. Decana de la Facultad de Ciencias Administrativas, Universidad de Lima- Perú. Docente universitaria y consultora.
} 


\begin{abstract}
Corporate social responsibility (CSR) is defined as the practices of the company based on ethical values and principles of transparency, which seek to promote the continuous improvement of the relationship between the company and its stakeholders (stakeholders). These segments include its customers, suppliers, partners, customers, the environment, communities, government and society in general. Thus, CSR comprises a wide range of voluntary activities of companies that aim to improve social and environmental performance of them and optimize their competitiveness. The application of Corporate Social Responsibility (CSR) in business has effects on organizational climate, operational performance, innovation and reputation of the companies. The incorporation of SMEs that are part of the value chain of large companies, in implementing CSR, will result in improved operational processes, streamlining of these processes, cost reduction and improved performance environmentally. The large and medium enterprises are responsible to include SMEs in the projects and activities mentioned, given the financial capacity of small businesses, the costs in training and certification would not be viable model of CSR. This application of CSR, however, presents a number of strategic and operational challenges that can be seen as opportunities for development and improvement of business, which require managers to be more creative and responsible, to combine a vision of the company focused on increasing profits and increasing activities on behalf of its suppliers, customers and society. The proposed strategy should encourage and facilitate companies in the textile and clothing sector to generate more jobs, best with large companies and relate to each other, incorporate the best and latest technology, to adapt to the new model of flexible specialization, can use easily financial and nonfinancial services offered to the sector, increase their creativity and generate technological innovations and improve their competitiveness.
\end{abstract}

Keywords: Competitiveness; corporate social responsibility; value chain.

\section{INTRODUCCIÓN}

La gestión de la cadena de valor de las grandes empresas se transforma en un elemento clave para la competitividad global. Las pymes que son proveedoras en esta cadena deben adoptar a la responsabilidad social empresarial (RSE) como una herramienta de gestión si desean mantenerse o ingresar a esta cadena, aumentando sus ingresos (como resultado de un mejor acceso al mercado, precios preferenciales) y reduciendo sus costos. Esta aplicación de la RSE presenta, no obstante, una serie de desafíos estratégicos y operativos que pueden considerarse como oportunidades de desarrollo y mejora de los negocios, las cuales obligan a los gerentes a ser más creativos y responsables, a combinar una visión de la empresa focalizada en el aumento de las utilidades y a incrementar las actividades en beneficio de sus proveedores, clientes y sociedad.

El presente trabajo, que se realizó en el período comprendido entre los años 2013 al 2014, tiene como objetivo de investigación demostrar que la responsabilidad social es una herramienta estratégica de la gran empresa del sector textil y confecciones y que aplicada a las pymes dentro de su cadena de valor, incrementará el nivel de competitividad en conjunto. El contexto espacial está conformado por las pymes textiles, las grandes empresas que las contratan para ser parte de su cadena de valor, asimismo a los representantes de los anteriores mencionados, como también al Estado mediante los organismos competentes.

\section{Planteamiento del estudio}

La sociedad tiene diferentes expectativas con relación a las empresas y es precisamente el concepto de la responsabilidad social empresarial (RSE) que comprende las motivaciones económicas, jurídicas, éticas y discrecionales que la sociedad manifiesta -en un momento determinado- sobre ellas. La noción de responsabilidad social descansa en la responsabilidad económica de la empresa (llamada también la perspectiva del valor para el accionista), es decir, está en la base $y$ es el fundamento sobre el cual se cimientan las otras responsabilidades empresariales. Las responsabilidades legales, obedecer las leyes y normas sociales respecto a lo permitido y no permitido, "jugar dentro de las reglas", van en un segundo nivel. Las nociones basadas en el modelo del "stakeholder" son superiores, en el sentido de que reconocen que las obligaciones éticas y morales van más allá del simple cumplimiento de las "reglas del juego" y comprenden la obligación de hacer lo justo, evitar los daños y contribuir con recursos para mejorar la calidad de vida de la comunidad. De esta manera, los gerentes están 
frente a un nuevo reto, el cual es más exigente que el impuesto por la filosofía de la calidad total en la década de 1980. Este reto es el de crear valor de una manera sostenible, sin provocar daños irreparables a la sociedad y el ambiente: ser socialmente responsables. El enfoque de la RSE aparece entonces como un medio de crear valor en una empresa para todos sus actuantes, los accionistas ("shareholders") y los llamados stakeholders: clientes, directivos, trabajadores, gobiernos locales, entre otros. Ello determina tener en cuenta que en la responsabilidad social empresarial existen dos dimensiones: una dimensión interna que comprende el ambiente de trabajo laboral, el acceso a capacitación y perfeccionamiento, condiciones laborales, etc.; y una dimensión externa que involucra el manejo del medio ambiente, cultura, salud, entre otros.

Belaunde, Parodi y Muñoz señalan:

“... podemos sostener que la responsabilidad social es una filosofía y un modo de actuación que va más allá de la generación de utilidades en el corto plazo, pues busca una relación más integral y estable de la empresa con su entorno interno (trabajadores) y externo (comunidad en la que se inserta). Es una manera de proceder de la empresa vinculada con el respeto a los valores éticos en concordancia con el cumplimiento de requerimientos $u$ obligaciones legales, respeto por las personas, por la comunidad en la que se insertan y por el medio ambiente".

Las empresas, conforme se internacionalizan, tienen que tomar decisiones respecto de la localización de sus mercados, sus plantas industriales, laboratorios de investigación y outsourcing, entre otros. Esta circunstancia las lleva a discernir sobre las características de los ambientes de negocio de los países o regiones, evaluar factores como la calidad de la mano de obra, infraestructura, cantidad y calidad de proveedores; es decir las dimensiones del enfoque de la responsabilidad social empresarial.

La ventaja competitiva de una empresa no puede ser explicada como un todo en la organización, sino que precisa de un análisis exhaustivo para comprender cuáles son las actividades que generan valor en la empresa. A través de la cadena de valor se analizan de forma sistémica las actividades de una empresa y se evalúan las fuentes de valor.
Los conceptos de competitividad y responsabilidad social empresarial van de la mano y comprenden a todas las empresas, especialmente las pequeñas y medianas empresas que tienen una relevancia creciente en la creación del empleo y el desarrollo de los países de América Latina. Estas empresas se engarzan en el mercado global a través de la tercerización o siendo parte de la cadena de valor de las grandes empresas y requieren mejorar su competitividad e incrementar sus oportunidades de mercado. La cadena de valor está conformada por una secuencia de factores de producción orientados para la consecución de un objetivo. Estos factores se combinan para crear un producto o servicio transable desde su concepción hasta el consumo final. Esta secuencia incluye a actividades como el diseño, la producción, el marketing, la distribución y los servicios de soporte al consumidor final. La cadena de valor es una metodología utilizada para analizar y desarrollar clúster o sectores, basada en el supuesto de que el desempeño económico y la competitividad de los clúster o sectores dependen de cómo se relacionan con otros actores que participan de la cadena de valor. La mejora del clúster se refiere a un proceso multidimensional que ayuda a elevar la competitividad de las empresas y sus clúster creando al mismo tiempo un impacto positivo en los trabajadores, la comunidad y la sociedad en su conjunto.

Metodologías para la aplicación de actividades de responsabilidad social empresarial (RSE) en las pymes

A la luz de la literatura examinada, se define a la RSE como un conjunto de políticas, prácticas y programas que se incorporan en todos los procesos de toma de decisiones de las empresas y ha venido siendo desarrollado, en primer lugar, por las grandes empresas transnacionales como parte de la cultura de competencia global.

La implementación de estas prácticas en las grandes empresas tiene un doble efecto:

i) Aumenta su competitividad regional y global incrementando su productividad y creándole una imagen positiva que impacta tangiblemente en sus accionistas, empleados, consumidores, proveedores y la sociedad en general.

ii) Tiene un efecto positivo en la competitividad de las pequeñas y medianas empresas (pymes) que conforman su cadena de valor. 
La gestión de la cadena de valor de las grandes empresas se transforma en un elemento clave para la competitividad global. Las pymes que son proveedoras en esta cadena, deben adoptar a la RSE como una herramienta de gestión si desean mantenerse o ingresar a esta cadena, aumentando sus ingresos (como resultado de un mejor acceso al mercado, precios preferenciales) y reduciendo sus costos.

Existen varios métodos para garantizar y evaluar la RSE de las empresas que pueden ser aplicadas a las pymes como, por ejemplo, el método TEAR del Instituto ETHOS del Brasil, el método Account Ability 1000 y la norma SA8000. En el 2003, el Instituto ETHOS y el Servicio Brasileño de Apoyo a las Micro y Pequeñas Empresas (Sebrae) publicaron dos herramientas a fin de estimular la incorporación de la RSE a las pymes: Herramienta de Autoevaluación y Planificación-Indicadores ETHOS-Sebrae de RSE para Micro y Pequeñas Empresas y Responsabilidad Social y Empresarial para Micro y Pequeñas Empresas-Paso a Paso. Estas herramientas se basan en la concepción de que el mercado está valorando otro vértice de la calidad: la calidad de las relaciones, es decir relaciones éticas y transparentes con todos los involucrados en el ambiente de negocio de las pymes.

\section{Objetivos de la investigación}

Para la presente investigación se plantearon los siguientes objetivos de trabajo:

\section{Objetivo general}

Demostrar que la responsabilidad social como herramienta estratégica de la gran empresa del sector textil y confecciones, aplicada a las PYMES dentro de su cadena de valor incrementara el nivel competitividad en conjunto.

\section{Objetivos específicos}

- Demostrar que las grandes empresas del sector textil y confecciones aplican la RSE como una estrategia.

- Demostrar que las PYMES del sector textil y confecciones adoptan la RSE como una herramienta estrategia para incrementar su competitividad.

\section{Gráfico N․o 1}

Factores internos y de entorno que inciden en las pymes.

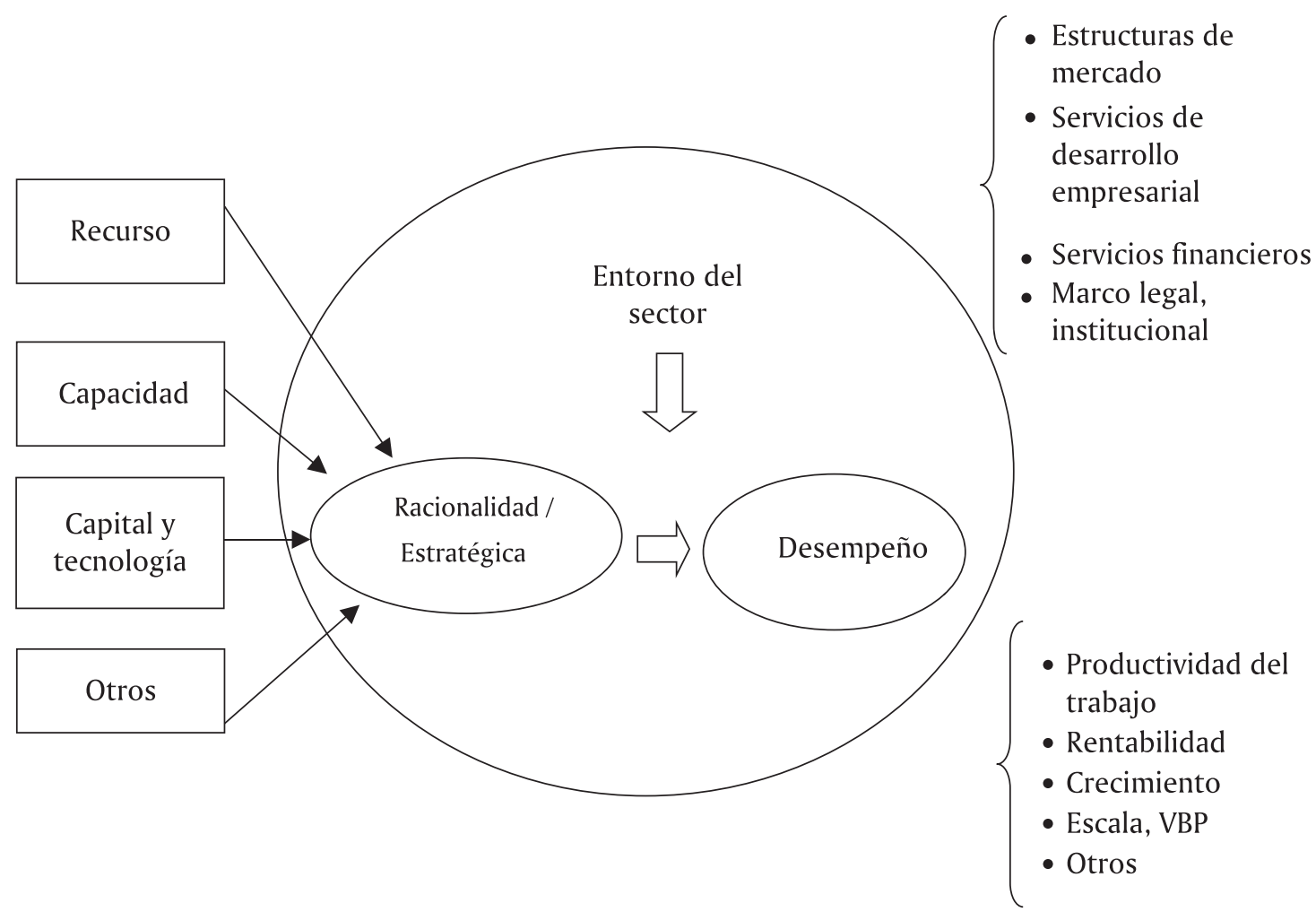

Fuente: Elaboración propia 
- Explicar la relación que existe entre los niveles de competitividad de las grandes empresas y las PYMES que integran de su cadena de valor, en el sector textil y confecciones.

\section{Hipótesis demostrada}

De la misma forma se planteó la siguiente hipótesis general:

\section{Hipótesis General}

La responsabilidad social empresarial utilizada como herramienta estratégica de la gran empresa del sector textil y confecciones, aplicada a las PYMES dentro de su cadena de valor SI incrementará el nivel competitividad en conjunto.

Hipótesis Específicas

Hipótesis Específica a

Existe una variación en la aplicación de los principios de la RSE, como estrategia por parte de la gran empresa del sector textil y confecciones.

\section{Hipótesis Específica b}

Las PYMES del sector textil y confecciones sí utilizan la RSE como una herramienta estratégica para incrementar su competitividad.

\section{Hipótesis Específica c}

Sí existe una relación directa entre los niveles de competitividad de las grandes empresas y las PYMES que integran de su cadena de valor, en el sector textil y confecciones.

\section{Método}

Este trabajo se centra en la demostración de la relación existente entre los niveles de competitividad de las pymes del sector industrial de los textiles y las confecciones que pertenecen a la cadena de valor de las grandes empresas de este sector y la aplicación de estrategias de la responsabilidad social empresarial (RSE).

1

2

2.1

2.2

\section{3}

La presente investigación se aplica a las pymes de la industria textil y confecciones, del distrito de La Victoria, y las grandes empresas de este sector que tercerizan la producción a las pequeñas empresas que conforman nuestra primera unidad de análisis. Estas unidades de análisis nos brindarán la información requerida para cumplir los objetivos del estudio.

El universo de investigación está compuesto por todas las pymes del rubro textil y confecciones en Gamarra (La Victoria). La población es conocida con un total de 552 pymes, de la misma forma se complementa con las opiniones que emiten los gerentes y expertos con relación al nivel de competitividad de las grandes empresas del sector textil y confecciones.

La muestra se determinó de forma estadística enfatizando el conocimiento de la población total, trabajando con un nivel de confianza de $95 \%$, un error muestral de $+/-7.5 \%$ y una proporción muestral del $50 \%$. Para los fines de la presente investigación, se ha optado por asignarle las mismas probabilidades de ser tomados en la muestra. El tamaño de muestra para el presente estudio es de 131 unidades, que es igual a 131 pymes textiles ubicadas en el distrito de La Victoria. Se realizó un muestreo no probabilístico, y se escogió dentro de los diferentes métodos, el muestreo por cuotas o accidental.

\section{Recolección e interpretación de datos}

Se aplicó el método Delphi con expertos que forman parte de la industria textil peruana en la gran empresa. Los resultados mostraron que consideran como herramientas estratégicas para incrementar la competitividad de las pymes que forman parte de su cadena de valor:

\section{- $\quad$ Formalización de las pymes}

El $60 \%$ de los expertos están de acuerdo y el $20 \%$ en total acuerdo en que la pyme se encuentra ubicada en la actividad primaria de producción de la cadena de valor de la gran empresa del sector textil y confecciones a través de las actividades de outsourcing. Lo que significa que al ser parte de la cadena de valor, existe efecto en la gran empresa si la pyme tiene algún cambio. La formalización de la pyme es beneficiosa para esta, ya que permite que se desarrolle y pueda crecer como empresa; si la gran em- 
presa textil brinda capacitación a las pymes en actividades de RSE, llega a obtener un efecto positivo en la competitividad.

\section{- $\quad$ Capacitación a las pymes}

Del total de los participantes del Delphi, el $60 \%$ están totalmente de acuerdo en que una mejora en la competitividad de las pymes, que forman parte de su cadena de valor, contribuye directamente a una mejora en los niveles de competitividad de la gran empresa, y el otro $40 \%$ está de acuerdo que tiene una contribución directa, es decir el total admite la relación de beneficio directa entre la pyme y la gran empresa. La formalización es un factor directo para el incremento de competitividad de la pyme, lo que significaría un incremento directo a la competitividad de la gran empresa a causa de la relación directa.

A pesar de aceptar esta relación directa y del beneficio que existe, la gran empresa no usa herramientas como la capacitación para promover la competitividad. El $100 \%$ de los participantes concuerdan que la gran empresa del sector textil y confecciones no tiene y ejecuta metodologías para la capacitación de las pymes, que forman parte de su cadena de valor, basados en los principios de responsabilidad social empresarial (RSE).

\section{- $\quad$ Financiamiento a las pymes}

Dentro del factor de financiamiento de las pymes, nos interesa conocer si la ayuda en financiamiento a las pymes por parte de la gran empresa textil, incrementa su competitividad y da beneficio a la gran empresa.

Esto es importante, ya que si el socio estratégico mejora su competitividad la otra parte se verá beneficiada; ejemplo de ello es la optimización en infraestructura de las pymes, que permite mejorar en su competitividad, la mejora en infraestructura se apoya con el cofinanciamiento de la gran empresa hacia las pymes por temas de facilitación, estas políticas de RSE ayudan al progreso de la pyme como socio estratégico.

El $80 \%$ de los expertos del sector textil y confecciones estuvieron de acuerdo y en total acuerdo, tal y como se aprecia en el gráfico, que estas políticas de RSE hacia las pymes les permiten mejorar sus relaciones con sus socios empresariales e inversores, así como mejorar sus resultados económicos.

\section{- $\quad$ Entorno social de las pymes}

Dentro del factor de entorno social, nos interesa conocer si la RSE influye en el entorno social de las pymes. El $40 \%$ de los expertos indican que en el marco de la RSE, la gran empresa del sector textil y confecciones es cada vez más exigente con el nivel de compromiso social y medioambiental de las pymes que forman parte de su cadena de valor, mientras que el $60 \%$ tiene la postura de indeciso sobre este tema. La aplicación de RSE no solo beneficia positivamente a la empresa con relación a la productividad de la pyme, sino que ayuda a la gran empresa textil en su imagen en la sociedad, es decir en la aceptación por parte de la población. El $100 \%$ de los expertos están de acuerdo y totalmente de acuerdo en que la implementación de actividades de RSE en la gran empresa del sector textil y confecciones incrementa su productividad y le crea una imagen positiva que impacta tangiblemente en sus stakeholders.

El $62.50 \%$ de los funcionarios del sector textil consultados indicaron que estuvieron en total acuerdo y de acuerdo, con el hecho de que la empresa tiene prácticas de gestión que benefician el desarrollo de la comunidad, tales como: contratar empleados que vivan en los alrededores, adquirir productos y servicios de emprendedores locales, entre otros. De lo expuesto podemos interpretar el alto nivel de interés de las entidades consultadas por brindar un beneficio a las personas que forman parte de su círculo de influencia, hecho que caracteriza a las empresas con prácticas de responsabilidad social empresarial.

Respecto al papel de la gran empresa respecto a las pymes de su cadena de valor, el $60 \%$ de los expertos consultados indica que el principal factor que determina un incremento de la productividad y calidad de los procesos de producción en las pymes del sector textil y confecciones es el compromiso y liderazgo por parte de la gran empresa hacia la pyme. Una buena gestión de las pymes involucra beneficio directo para la gran empresa, debido a la relación en la cadena de valor como actividad primaria (ver gráfico $\mathrm{N} .^{\circ} 2$ ). 
Gráfico N.o 2: Factor capacitación de las pymes

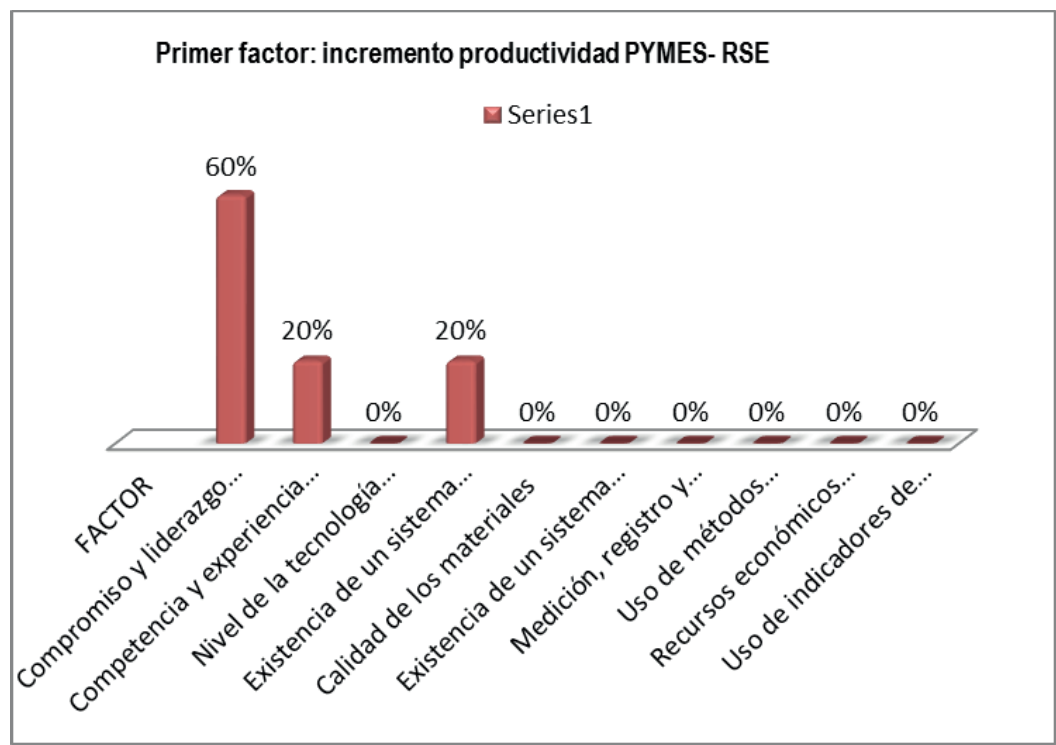

Fuente: Elaboración propia

\section{Comprobación de hipótesis}

Para comprobar si existe una variación entre el 2012 y el 2014 en la aplicación de los principios de Responsabilidad Social Empresarial como estrategia por parte de la gran empresa del Sector Textil y Confecciones, fue necesario aplicar la Prueba de Hipótesis, a través del método: P.H. de Independencia de Variables Chi-Cuadrado con un Nivel de Significación del 5\%.

Se obtuvo un Valor Crítico de 9.488, para una Chi-Cuadrado de 4 Grados de Libertad; mientras que el Valor Calculado para los datos obtenidos fue de 156.63 .

Por tanto, la hipótesis que postula que "SI existe una variación en la aplicación de los principios de la RSE, como estrategia por parte de la gran empresa del sector textil y confecciones", se acepta con mucha fuerza.

Respecto a la hipótesis: "Las PYMES del sector textil y confecciones SI utilizan la RSE como una herramienta estratégica (Formalización de las PYMES) para incrementar su competitividad".

El objetivo es tratar de comprobar si la formalización, como una variable de análisis para la elaboración de un modelo de gestión de la RSE de las grandes empresas de la industria textil - confecciones, aborda el cumplimiento por las PYMES de las normas que regulan la actividad empresarial que desempeñan. Para ello fue necesario aplicar la Prueba de Hipótesis, a través del método: P.H. de Independencia de Variables Chi-Cuadrado con un Nivel de Significación del 5\%.

\begin{tabular}{ccccc}
\hline \multirow{2}{*}{$\begin{array}{c}\text { RESPONSABILIDAD SOCIAL } \\
\text { EMPRESARIAL }\end{array}$} & \multicolumn{4}{c}{ FORMALIZACIÓN DE LAS PYMES } \\
\cline { 2 - 5 } & Total acuerdo & De acuerdo & $\begin{array}{c}\text { Indeciso-Desacuerdo } \\
\text { - Total desacuerdo }\end{array}$ & \\
\hline & \multicolumn{2}{c}{ ESTADÍSTICO DE LA PRUEBA } \\
\hline Total acuerdo & 22.48 & 5.94 & 8.57 & 36.98 \\
\hline De acuerdo & 16.15 & 20.50 & 5.97 & 42.62 \\
\hline $\begin{array}{c}\text { Indeciso - Desacuerdo } \\
\text { - Total Desacuerdo }\end{array}$ & 6.67 & 6.27 & 85.68 & 98.62 \\
\hline TOTAL & 45.30 & 32.70 & 100.21 & 178.21 \\
\hline
\end{tabular}


Función de Probabilidad Chi - Cuadrado 4 con Grados de Libertad:

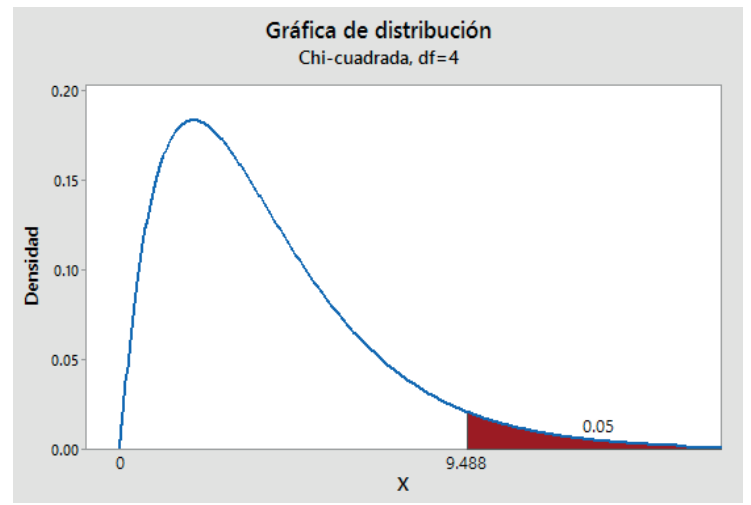

Como podemos apreciar en la gráfica arrojada por el Software MINITAB 17, nuestro $X_{4}^{2}=178.21$ es bastante mayor al Valor Crítico $X_{(4 ; 0.95)}^{2}=9.488$, es decir, pertenece a la Región de Rechazo; por lo que se procede a rechazar $H_{0}$. Decisión: Se acepta $H_{1}$ Se obtuvo un Valor Crítico de 9.488, para una Chi-Cuadrado de 4 Grados de Libertad; mientras que el Valor Calculado para los datos obtenidos fue de 178.21.

Respecto a la hipótesis si las capacitaciones, realizadas en las PYMES, se efectúan con la finali- dad de incrementar el conocimiento, las habilidades o modificar las conductas de los trabajadores ( partiendo del hecho que existe una diferencia entre el conocimiento que requiere la función que desempeñan y el conocimiento que realmente poseen), se aplica la Prueba de Hipótesis, a través del método: P.H. de Independencia de Variables Chi-Cuadrado con un Nivel de Significación del 5\%.

Se obtuvo un Valor Crítico de 9.488, para una Chi-Cuadrado de 4 Grados de Libertad; mientras que el Valor Calculado para los datos obtenidos fue de 178.21. Por tanto, la hipótesis que postula que "Las PYMES del sector textil y confecciones SI utilizan la RSE como una herramienta estratégica (Capacitación a las PYMES) para incrementar su competitividad", se acepta con mucha fuerza.

Para comprobar la hipótesis sí el financiamiento, como una variable de análisis para la elaboración de un modelo de gestión de la RSE de las grandes empresas de la industria textil - confecciones, aborda el tema de los recursos financieros entregado a las PYMES para desempeñar su actividad económica en mejores condiciones, fue necesario aplicar la Prueba de Hipótesis, a través del método: P.H. de Independencia de Variables Chi-Cuadrado con un Nivel de Significación del 5\%.

Tabla de Contingencia: Prueba de hipótesis Chicuadrado de independencia de variables.

\begin{tabular}{cccc}
\hline \multirow{2}{*}{$\begin{array}{c}\text { RESPONSABILIDAD SOCIAL } \\
\text { EMPRESARIAL }\end{array}$} & Total acuerdo & $\begin{array}{c}\text { Feacuerdo-Indeciso-Desacuerdo } \\
- \text { - Total desacuerdo }\end{array}$ & TOTAL \\
\cline { 2 - 4 } & 46 & VALORES OBSERVADOS & \\
\hline Total acuerdo & 32 & 20 & 66 \\
\hline De acuerdo & 0 & 14 & 46 \\
\hline $\begin{array}{c}\text { Indeciso - Desacuerdo } \\
\text { - Total Desacuerdo }\end{array}$ & 78 & 19 & 19 \\
\hline TOTAL & 39.30 & VALORES ESPERADOS & 66 \\
\hline Total acuerdo & 27.39 & 26.70 & 46 \\
\hline De acuerdo & 11.31 & 18.61 & 19 \\
\hline $\begin{array}{c}\text { Indeciso - Desacuerdo } \\
\text { - Total Desacuerdo }\end{array}$ & 78 & 7.69 & 131 \\
\hline TOTAL & & 53 & \\
\hline
\end{tabular}


Estadístico de la Prueba

\begin{tabular}{cccc}
\hline \multirow{2}{*}{$\begin{array}{c}\text { RESPONSABILIDAD SOCIAL } \\
\text { EMPRESARIAL }\end{array}$} & \multicolumn{2}{c}{ FINANCIAMIENTO A LAS PYMES } & \\
\cline { 2 - 3 } & Total acuerdo & $\begin{array}{c}\text { Deacuerdo-Indeciso-Desacuerdo } \\
\text { - Total desacuerdo }\end{array}$ & TOTAL \\
\hline Total acuerdo & 1.14 & 1.68 & 2.83 \\
\hline De acuerdo & 0.78 & 1.14 & 1.92 \\
\hline $\begin{array}{c}\text { Indeciso - Desacuerdo } \\
\text { - Total Desacuerdo }\end{array}$ & 11.31 & 16.65 & 27.96 \\
\hline TOTAL & 13.23 & 19.47 & 32.71 \\
\hline
\end{tabular}

Se obtuvo un Valor Crítico de 5.991, para una Chi-Cuadrado de 2 Grados de Libertad; mientras que el Valor Calculado para los datos obtenidos fue de 32.71. Por tanto, la hipótesis $\mathrm{H} 1$, que postula que "Las PYMES del sector textil y confecciones SI utilizan la RSE como una herramienta estratégica (Financiamiento a las PYMES) para incrementar su competitividad", se acepta con mucha fuerza.

Para comprobar sí el entorno social, como una variable de análisis para la elaboración de un modelo de gestión de la RSE de las grandes empresas de la industria textil - confecciones, aborda el tema de la responsabilidad y la ética aplicada por las PYMES en la relación con ambiente de desarrollo, lo cual incluye las familias de sus trabajadores, locales y viviendas colindantes a sus instalaciones y el medio ambiente en general, fue necesario aplicar la Prueba de Hipótesis, a través del método: P.H. de Independencia de Variables Chi-Cuadrado con un Nivel de Significación del 5\%.

Se obtuvo un Valor Crítico de 9.488, para una Chi-Cuadrado de 4 Grados de Libertad; mientras que el Valor Calculado para los datos obtenidos fue de 97.24. Por tanto, la hipótesis que postula

Tabla de Contingencia: Prueba de hipótesis Chicuadrado de independencia de variables

\begin{tabular}{|c|c|c|c|c|}
\hline \multirow{2}{*}{$\begin{array}{c}\text { RESPONSABILIDAD SOCIAL } \\
\text { EMPRESARIAL }\end{array}$} & \multicolumn{3}{|c|}{ MEJORA EL ENTORNO SOOCIAL DE LAS PYMES } & \multirow[b]{2}{*}{ TOTAL } \\
\hline & Total acuerdo & De acuerdo & $\begin{array}{l}\text { Indeciso- Desacuerdo } \\
\text { - Total desacuerdo }\end{array}$ & \\
\hline & \multicolumn{3}{|c|}{ VALORES OBSERVADOS } & \\
\hline Total acuerdo & 46 & 2 & 18 & 66 \\
\hline De acuerdo & 20 & 27 & 0 & 47 \\
\hline $\begin{array}{l}\text { Indeciso - Desacuerdo } \\
\text { - Total Desacuerdo }\end{array}$ & 1 & 0 & 17 & 18 \\
\hline \multirow[t]{2}{*}{ TOTAL } & 67 & 29 & 35 & 131 \\
\hline & \multicolumn{3}{|c|}{ VALORES ESPERADOS } & \\
\hline Total acuerdo & 33.76 & 14.61 & 17.63 & 66 \\
\hline De acuerdo & 24.04 & 10.40 & 12.56 & 47 \\
\hline $\begin{array}{l}\text { Indeciso - Desacuerdo } \\
\text { - Total Desacuerdo }\end{array}$ & 9.21 & 3.98 & 4.81 & 18 \\
\hline TOTAL & 67 & 29 & 35 & 131 \\
\hline
\end{tabular}

Estadístico de la Prueba

\begin{tabular}{ccccc}
\hline \multirow{2}{*}{$\begin{array}{c}\text { RESPONSABILIDAD SOCIAL } \\
\text { EMPRESARIAL }\end{array}$} & \multicolumn{4}{c}{ MEJORA EL ENTORNO SOCIAL DE LAS PYMES } \\
\cline { 2 - 5 } & $\begin{array}{c}\text { Total acuerdo } \\
\text { ESTADÍSTICO DE LA PRUEBA }\end{array}$ & De acuerdo & $\begin{array}{c}\text { Indeciso-Desacuerdo } \\
\text { - Total desacuerdo }\end{array}$ & \\
\hline Total acuerdo & 4.44 & 10.88 & 0.01 & 15.33 \\
\hline De acuerdo & 0.68 & 26.47 & 12.56 & 39.71 \\
\hline $\begin{array}{c}\text { Indeciso - Desacuerdo } \\
\text { - Total Desacuerdo }\end{array}$ & 7.32 & 3.99 & 30.90 & 42.20 \\
\hline TOTAL & 12.43 & 41.34 & 43.47 & 97.24 \\
\hline
\end{tabular}


que "Las PYMES del sector textil y confecciones SI utilizan la RSE como una herramienta estratégica (Entorno Social de las PYMES) para incrementar su competitividad", se acepta con mucha fuerza.

Para comprobar si las herramientas estratégicas empleadas por la PYME (formalización, Capacitación, Financiamiento y Entorno Social) contribuyen a una mejora en su competitividad, fue necesario aplicar la Prueba de Hipótesis, a través del método: P.H. de Independencia de Variables Chi-Cuadrado con un Nivel de Significación del 5\%.

Se obtuvo un Valor Crítico de 5.991, para una Chi-Cuadrado de 2 Grados de Libertad; mientras que el Valor Calculado para los datos obtenidos fue de 116.28. Por tanto, la hipótesis H1, que postula que "Las PYMES del sector textil y confecciones SI utilizan la RSE como una herramienta estratégica para incrementar su competitividad", se acepta con mucha fuerza.

\section{Incremento de la competitividad en las pymes}

Tal y como se aprecia en las pruebas de hipótesis realizadas, ya se ha demostrado que las pymes del sector textil y confecciones sí utilizan la responsabilidad social empresarial como una herramienta estratégica para incrementar su competitividad.

Estos resultados se integran con los obtenidos por Bonilla Pastor en su tesis titulada: "La gestión de la calidad en las mypes de la confección textil de Lima y Callao y su relación con los costos generados por desechos y desperdicios". En el estudio realizado por Bonilla se aprecia una cuantificación económica de los desechos y desperdicios generados por cada mype. En términos económicos, la investigación de Bonilla muestra el valor de los desechos y desperdicios para luego relacionarlos con el costo de producción, para el periodo de un año (2012). Se observa que el costo de desechos y desperdicios en las mypes de la confección textil

Tabla de Contingencia: Prueba de hipótesis Chi-cuadrado de independencia de variables

\begin{tabular}{|c|c|c|c|}
\hline \multirow{2}{*}{$\begin{array}{c}\text { RESPONSABILIDAD SOCIAL } \\
\text { EMPRESARIAL }\end{array}$} & \multicolumn{2}{|c|}{ CONSOLIDADO HERRAMIENTAS ESTRATÉGICAS } & \multirow[b]{2}{*}{ TOTAL } \\
\hline & Total acuerdo & $\begin{array}{c}\text { Deacuerdo-Indeciso-Desacuerdo } \\
\text { - Total desacuerdo }\end{array}$ & \\
\hline \multicolumn{4}{|c|}{ VALORES OBSERVADOS } \\
\hline Total acuerdo & 62 & 0 & 62 \\
\hline De acuerdo & 4 & 46 & 50 \\
\hline $\begin{array}{l}\text { Indeciso - Desacuerdo } \\
\text { - Total Desacuerdo }\end{array}$ & 0 & 19 & 19 \\
\hline TOTAL & 66 & 65 & 131 \\
\hline \multicolumn{4}{|c|}{ VALORES ESPERADOS } \\
\hline Total acuerdo & 31.24 & 30.76 & 62 \\
\hline De acuerdo & 25.19 & 24.81 & 50 \\
\hline $\begin{array}{l}\text { Indeciso - Desacuerdo } \\
\text { - Total Desacuerdo }\end{array}$ & 9.57 & 9.43 & 19 \\
\hline TOTAL & 66 & 65 & 131 \\
\hline
\end{tabular}

Estadístico de la Prueba

\begin{tabular}{cccc}
\hline \multirow{2}{*}{$\begin{array}{c}\text { RESPONSABILIDAD SOCIAL } \\
\text { EMPRESARIAL }\end{array}$} & CONSOLIDADO HERRAMIENTAS ESTRATÉGICAS & \\
\cline { 2 - 3 } & Total acuerdo & $\begin{array}{c}\text { Deacuerdo-Indeciso-Desacuerdo } \\
\text { - Total desacuerdo }\end{array}$ & TOTAL \\
\hline Total acuerdo & 30.30 & 30.76 & 61.06 \\
\hline De acuerdo & 17.83 & 18.10 & 35.93 \\
\hline $\begin{array}{c}\text { Indeciso - Desacuerdo } \\
\text { - Total Desacuerdo }\end{array}$ & 9.57 & 9.72 & 19.29 \\
\hline TOTAL & 57.70 & 58.58 & 116.28 \\
\hline
\end{tabular}


de prendas, representa en promedio un $7.6 \%$, siendo el valor mínimo de $5 \%$ y el mayor de 11 $\%$; cifras significativas para empresas que tienen limitaciones económicas. Compañías líderes en el sector de la confección promedian el $2 \%$.

Con este análisis se demostró el postulado de que: "Mientras mejor sea la gestión de la calidad en las mypes de la confección textil de Lima y Callao , que elaboran prendas con tejido de punto o tejido plano, menor será el nivel de los costos generados por desechos y desperdicios"; considerando que la responsabilidad social empresarial contribuye a una gestión de calidad, se refuerza que: "Las pymes del sector textil y confecciones sí utilizan la RSE como una herramienta estratégica para incrementar su competitividad".

\section{Relación entre pyme y la gran empresa del sector textil y confecciones a través de la cadena de valor}

La relación entre las pymes y la gran empresa del sector textil y confecciones se da a través de la cadena de valor. Esta información se corrobora con los resultados del método Delphi aplicado el año 2014, donde se refleja la opinión de los expertos sobre si las pymes se encuentran ubicadas en la actividad primaria de producción de la cadena de valor de la gran empresa del sector textil y confecciones a través de las actividades de outsourcing. El $80 \%$ indica que están de acuerdo y total acuerdo respecto que la pyme forma parte de la cadena de valor de la gran empresa del sector textil y confecciones; mientras que solo el $20 \%$ maneja una posición de indecisión si la pyme forma parte de la cadena de valor en las actividades primarias de la gran empresa del sector textil y confecciones, y el $0 \%$ indica estar en desacuerdo o total desacuerdo en que la relación entre las partes es por la cadena de valor.

\section{Relación directa entre pyme y gran empresa de sector textil y confecciones (beneficios)}

Se entiende que la disminución de las mermas por parte de las pymes es una prueba de la mejora en su eficiencia y productividad, en el aspecto de reducción de costos, teniendo el producto a niveles más competitivos; es decir, entramos a la mejora de la competitividad de la pyme. Hasta este punto se comprueba la mejora en competitividad por parte de la pyme, pero ha afectado en algo la competitividad en la gran empresa del sector textil y confecciones.
El incremento en los niveles de competitividad de las pymes podrá beneficiar a la gran empresa, si y solo si existe una relación directa entre las dos. Acorde con los expertos, en el Delphi indican que existe una relación directa entre las partes. En el gráfico se refleja la opinión de los expertos sobre la mejora directa a los niveles de competitividad de la gran empresa, a través de la cadena de valor, como resultado de la mejora a nivel competitivo de la pyme. El 60 \% está en total acuerdo sobre el efecto directo de competitividad entre las pymes y la gran empresa del sector textil y confecciones, además que el $40 \%$ está de acuerdo que si la pyme aumenta su competitividad, esta tendrá un efecto directo en la competitividad de la gran empresa del sector textil y confecciones gracias a la relación que exista entre las dos partes por las cadena de valor.

Además que existen beneficios para la gran empresa del sector textil y confecciones por la aplicación de actividades de RSE a las pymes. En la opinión de los expertos, el factor implementación de actividades de RSE en la gran empresa del sector textil y confecciones incrementa su productividad y le crea una imagen positiva que impacta tangiblemente en sus stakeholders.

Ante esto el $80 \%$ indica su total acuerdo sobre la mejora de productividad para la gran empresa ante la aplicación de actividades de RSE, además que el $20 \%$ restante está de acuerdo sobre el planteamiento de beneficio de la gran empresa textil y confecciones por la mejora de imagen ante sus stakeholders.

Con estas tres afirmaciones y secuencialidad, se comprueba que sí existe una relación directa entre los niveles de competitividad de las grandes empresas y las pymes que integran su cadena de valor, en el sector textil y confecciones.

\section{Conclusiones}

La responsabilidad social empresarial es un elemento integrante de la gestión, integrando los objetivos estratégicos con las actividades que impliquen acciones específicas, desarrolladas con criterios y políticas institucionales, de acuerdo con las peculiaridades de cada empresa. Según Sarmiento del Valle (2010), la responsabilidad social debe verse como una posibilidad de aprovechar oportunidades, desarrollando las competencias de sus dirigentes que gestionen adecuadamente recursos de las empresas con conciencia social y 
ambiental. Una gestión estratégica de la responsabilidad social es un costo de oportunidad que se refleja en el corto plazo, pero un factor de ganancias en el largo plazo.

El modelo propuesto de RSE es la conformación de alianzas estratégicas entre las grandes empresas y las pymes relacionadas con su cadena de valor. Esta alianza supondrá un beneficio mutuo y permitirá el aumento de la competitividad de ambas empresas por la vía de la introducción de mejoras con un enfoque RSE en alguna de las siguientes áreas: (i) gobierno de la empresa (ética y transparencia); (ii) gestión de los recursos humanos (calidad de vida laboral); (iii) relación con el cliente a través de políticas de calidad, de producto y de atención al cliente; (iv) relación con proveedores; y (v) relación con la comunidad. A esta alianza puede sumarse el apoyo de organizaciones no gubernamentales, organizaciones multilaterales y universidades.

Las grandes empresas que participarían de este modelo deben ser empresas nacionales líderes del sector textil, comprometidas a implementar actividades de RSE y que estén dispuestas a reclutar como mínimo diez pymes que integren su cadena. Las pymes seleccionadas por esta empresa ancla deben pertenecer al rubro de pequeña empresa, definida por el número de trabajadores (11-49); estar legalmente constituida; manifestar interés en cumplir la normativa ambiental y laboral. El objetivo de este modelo es poner en práctica medidas de RSE en las pymes engarzadas a lo largo de la cadena de valor de una gran empresa textil, incluidos los proveedores principales y secundarios, los distribuidores y los puntos de venta final. Para la implementación de RSE en estas pymes, el modelo pretende que cada gran empresa contribuya con recursos financieros por cada pyme participante, asumiendo así una parte significativa para la implementación de RSE en las pymes de su cadena de valor. El modelo propuesto está basado en que la empresa clave asume a la
RSE como un compromiso de la alta dirección, lo que implica que esta debe incluir los principios de la RSE en la misión y los valores de la empresa y determinar las funciones de la responsabilidad social empresarial en su estructura organizacional. A partir de este paso las políticas y estrategias de la empresa incluyen a la RSE, lo que se reflejará en las políticas de recursos humanos, medio ambiente, relaciones con proveedores, etc. De esta manera, los procesos y procedimientos de la empresa se alinearán con las orientadas a la RSE (ver gráfico $\mathrm{N}{ }^{\circ} 8$ ).

Este alineamiento se traducirá en proyectos y actividades de RSE:

a. Inversión en RSE: asignación de recursos materiales, humanos y financieros para capacitación y difusión de RSE.

b. Proyectos sociales

c. Proyectos ambientales

La aplicación de la RSE en la empresa ancla tiene efectos en el clima organizacional, el desempeño operacional, la capacidad de innovación y en la reputación de la empresa. La incorporación de las pymes que forman parte de la cadena de valor de la empresa en la aplicación de la RSE se traducirá en una mejora de los procesos operativos, racionalización de estos procesos, reducción de costos y un mejor desempeño respecto al medio ambiente. Es la gran y mediana empresa la llamada a incluir a las pymes en los proyectos y actividades mencionados ya que dada la capacidad financiera de las pequeñas empresas frente a los costos en capacitación y certificación no sería viable el modelo de RSE. La estrategia propuesta debe incentivar y facilitar que las empresas del sector generen más empleo, que se relacionen mejor con las grandes empresas y entre sí, incorporen la mejor y más reciente tecnología, y generen innovaciones tecnológicas y mejoren su competitividad. 
Gráfico N. ${ }^{\circ} 8$

Enfoque propuesto

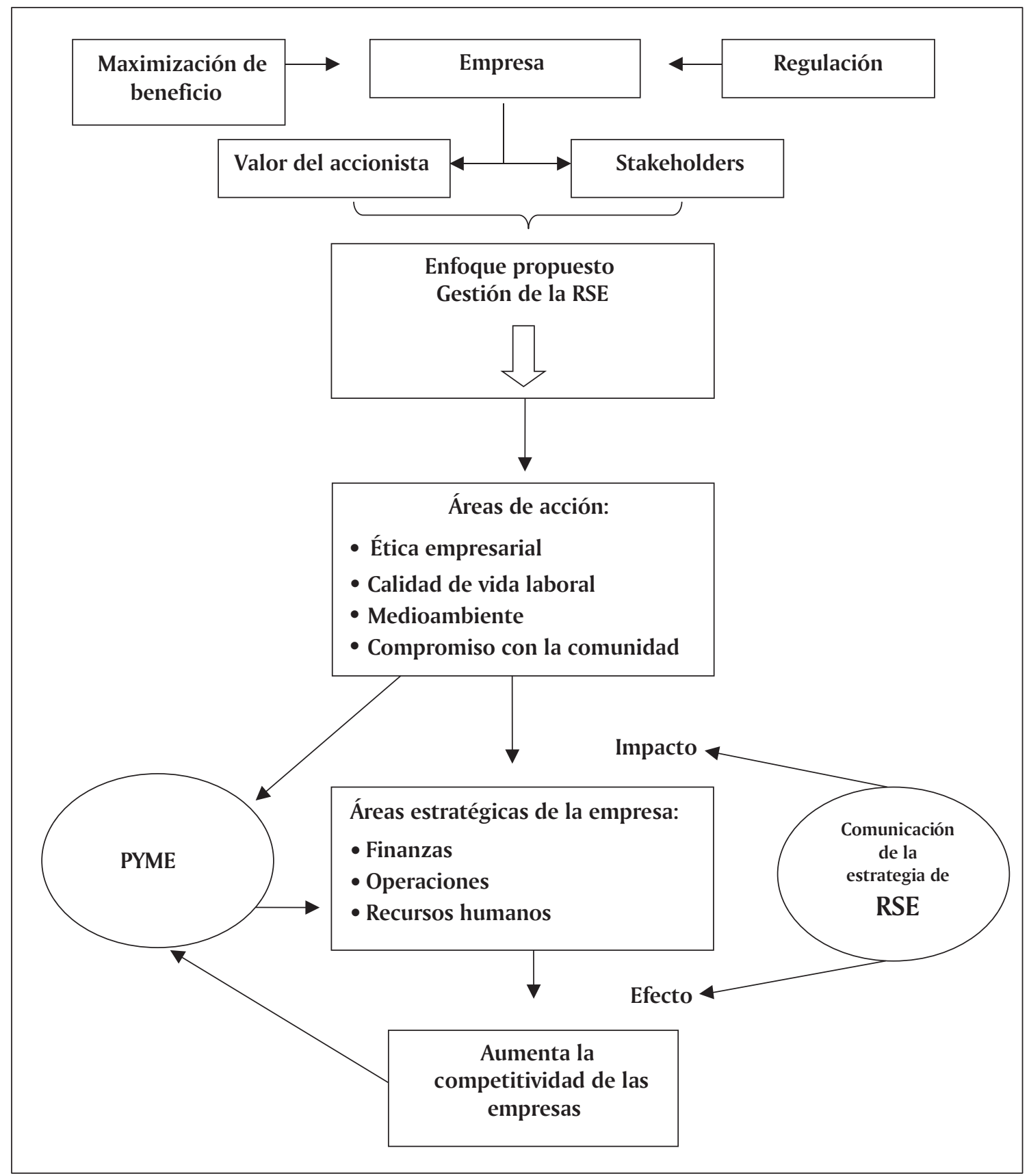

Fuente: Elaboración propia 


\section{Referencias bibliográficas}

Banco Interamericano de Desarrollo y Fondo Multilateral de Inversiones FOMIN. (2009). Guía de aprendizaje sobre la implementación de responsabilidad social empresarial en pequeñas y medianas empresas.

Bonilla, E. (2013). "La gestión de la calidad en las mypes de la confección textil de Lima y Callao y su relación con los costos generados por desechos y desperdicios". (Tesis doctoral). Universidad Nacional Mayor de San Marcos.

Cabrera, G. (2006). La responsabilidad social de la empresa y las Metas de Desarrollo del Milenio. MINEDU.

Cornejo, R. E. (2007). El desafío competitivo: Estrategias de empresas exportadoras peruanas exitosas. Lima: Editorial San Marcos.

De Belaunde, J., Parodi, B., \& Muñoz, D. (2001). Cómo promover la responsabilidad social empresarial en el Perú: Marco legal e institucional. Lima: Universidad del Pacífico, Centro de Investigaciones. Instituto Ethos de Em- presas e Responsabilidade Social. (2007). Metodología Tear de trabalho em cadeia de valor. Sao Paulo: Instituto Ethos.

Porter, M. E. (1991). La ventaja competitiva de las naciones. Buenos Aires: Javier Vergara

Rojas, V.J. (2013). Principales factores que impiden el crecimiento de las mypes, propuesta de estrategia para el desarrollo competitivo. (Tesis doctoral). Universidad Nacional Mayor de San Marcos.

Schwalb, M. M., Malca, O. (2004). Responsabilidad social: Fundamentos para la competitividad empresarial y el desarrollo sostenible. Lima: Universidad del Pacífico, Centro de Investigación.

Schwalb, M. M. (2004). Beneficios de la responsabilidad social empresarial y las inversiones socialmente responsables. Lima: Universidad del Pacífico, Centro de Investigación.

Vives, A., Corral, A., \& Isusi, I. (2005). Responsabilidad social de la empresa en las PYMEs de Latinoamerica. Washington: Banco Interamericano de Desarrollo. 